\title{
Multidimensional scaling of painful and innocuous electrocutaneous stimuli: Reliability and individual differences
}

\author{
MALVIN N. JANAL \\ New York State Psychiatric Institute, New York, New York \\ W. CRAWFORD CLARK \\ New York State Psychiatric Institute, New York, New York \\ and Columbia University, New York, New York \\ and \\ J. DOUGLAS CARROLL \\ Rutgers University, New Brunswick, New Jersey
}

\begin{abstract}
Multidimensional scaling was used to explore whether a single intensity dimension underlies the perception of both nonpainful and painful electrical stimuli, or whether separate dimensions are required. For the scaling (INDSCAL) procedure, 41 healthy volunteers judged the similarity between all pairs of 16 intensities, which ranged from imperceptible levels to pain tolerance. For the property mapping (PREFMAP) analysis, they rated each intensity on each of 16 property scales. INDSCAL revealed four dimensions that showed high levels of both test-retest and splithalf reliability. The first dimension scaled stimuli from the lowest intensity to the pain threshold. This dimension was related to property scales of sensation, affect, and arousal, but not pain, sug. gesting a sensory magnitude dimension. The second dimension ordered the stimuli from mildly to severely painful and was related to the painful property scale, suggesting a pain intensity dimension. Third and fourth dimensions, which refined the scaling of nonpainful stimuli, were also found. Variability in the subjects' use of the painful and nonpainful dimensions was related to their choice of stimulus descriptors. Like clinical pain, laboratory pain requires multidimensional assessment.
\end{abstract}

We (Clark, Carroll, Yang, \& Janal, 1986) previously used multidimensional scaling (MDS) to resolve the very old controversy with respect to the number of dimensions of thermal pain. On the major dimension of that study, the stimuli were ordered from weak to strong, while on the minor dimension, painful intensities were distinguished from innocuous (i.e., warm and hot) intensities. This two-dimensional solution demonstrated that pain was scaled on an independent dimension, rather than at the upper end of a single sensory strength continuum. In the present paper, we examine a similar question concerning the dimension(s) on which subjects categorize electrical stimuli in levels from innocuous to noxious. Is electrical pain the upper end of a sensory strength continuum, or does an independent pain dimension exist?

None of the methods widely used to study electrocutaneous sensibility, such as those of classical psychophysics, magnitude estimation, or signal detection theory, addresses the question of the number of dimensions under-

This study was supported in part by U.S. Public Health Service Grants NS-20248 and MH30906-06. Correspondence should be addressed to Malvin N. Janal, New York State Psychiatric Institute, 722 West 168th Street, New York, NY 10032. lying electrocutaneous judgments. In threshold models, it is assumed that one dimension, intensity, is sufficient, as was the case in early magnitude estimation studies (Stevens, Carton, \& Schickman, 1958). In later studies, it has been suggested that painful and nonpainful intensities follow different power functions (Rosner \& Goff, 1967) and that somatosensory pain and auditory pain also follow different power functions (Algom, Raphaeli, \& CohenRaz, 1986). Magnitude estimation studies of both sensory and affective intensity have been reported (Gracely, McGrath, \& Dubner, 1978), and three-dimensional models have also been suggested (Tursky, Jamner, \& Friedman, 1982). Finally, in studies based on signal detection theory, it is assumed that two indices, discriminability and response criterion, underlie the pain experience (Clark, 1974). Thus, these major psychophysical approaches disagree with respect to the number and nature of dimensions needed to account for pain perception. Furthermore, each method imposes the "dimensions," rather than allowing them to be discovered from subjects' reports (Clark, Janal, \& Carroll, 1989). By contrast, MDS methods are designed to discover the dimensions underlying subjective judgments (e.g., see Kruskal \& Wish, 1978; Schiffman, Reynolds, \& Young, 1981; Shepard, 
1980). By allowing the subject to use personal judgment criteria, unbiased by the experimenter's expectations about the number or type of dimensions, MDS affords the opportunity to discover the cognitive process that subjects use in the absence of explicit classification rules.

In addition to addressing experimenter (or method) bias effects, MDS is uniquely suited to the study of individual differences in subjective report. The individual differences scaling (INDSCAL) model (Arabie, Carroll, \& DeSarbo, 1987; Carroll, 1972; Carroll \& Chang, 1970), a threeway MDS model, provides a spatial representation of stimulus objects that is based on a weighted Euclidean metric. Subjects rate the similarity (more generally, the proximity) of all pairs of stimulus objects. From these proximities, the analysis constructs a group stimulus space of orthogonal dimensions. Relationships among stimulus objects on each dimension of this space are then used to infer the psychological distance between such objects. Individual differences are quantified in a subject (or source) space, which indexes the importance or salience to each subject of each of the group dimensions. These weights shrink or expand each dimension in proportion to its importance to each individual's judgments. Subject weights of zero, which indicate that a dimension is irrelevant to a subject's judgments, allow for group dimensions that are used only by a subsample of subjects.

MDS has been applied to the study of pain descriptors (Carasso, Yehuda, \& Mostofsky, 1984; Clark, FerrerBrechner, Janal, Carroll, \& Yang, 1989; Kwilosz, Green, \& Torgerson, 1983; Morley, 1989; Torgerson \& DenBebba, 1983) and pain coping strategies (Wack \& Turk, 1984). Only Clark et al. (1986), however, have applied MDS to the study of laboratory pain stimuli. We extend that study here by using electrical stimuli, by assessing test-retest reliability, and by relating indices of stimulus description to individual differences in the salience of each dimension. We also use property mapping (PREFMAP) here to aid in the interpretation of the dimensions. In this method, subjects rate each stimulus object on a variety of property scales expected to be relevant to the stimulus space by the experimenter. Regression models then relate vectors that are based on these ratings to each dimension of the group stimulus space (Carroll, 1972). Close associations between property vectors and dimensional axes are generally assumed to indicate that that scale was relevant to the subjects' judgments of similarity during the INDSCAL task. We test this assumption by also asking subjects to rate the relevance of the various properties to their similarity judgments.

\section{METHOD}

\section{Subjects}

Forty-one males, age 18-46 years $(M=28.0)$, who reported themselves free from current major illness and drug use, gave informed consent to a protocol approved by the local review board, and were paid for their participation. Twenty-five subjects returned for Day 2 reliability testing.
Materials

A DEC MINC-11/03 microcomputer controlled stimulus delivery and data acquisition in the method of limits, INDSCAL, and PREFMAP studies. Programming was by the first author in MINC BASIC.

A constant-voltage electrical stimulator (designed and built by M. Rosengarten, Chapel Hill, NC) delivered a 1-msec square-wave stimulus pulse at intensities up to $100 \mathrm{~V}$. A 12-bit D/A converter translated programmed pulse intensities into analog values between 0 and $10 \mathrm{~V}$. A series of operational amplifiers then multiplied these values by 10 to produce output stimuli from 0 to $100 \mathrm{~V}$ (in steps of $.024 \mathrm{~V}$ ). Each 1 -sec stimulus train delivered 10 pulses. The interpulse interval ( $99 \mathrm{msec}$ ) was controlled by a clock in the MINC, enabling and pulsing an analog output gate at appropriate intervals. A transformer isolated the subject from ground.

The intensity of each stimulus pulse was measured. The voltage across the subject's electrodes was stored by a sample/hold circuit and sampled (under software control) by a MINC A/D converter. A second A/D converter sampled the current, which was calculated from the small voltage drop through a 500 resistor connected in series with one of the electrodes. Voltage and current measures were used to calculate pulse power. Since the electrode-skin junction presents an unpredictable configuration of capacitance and impedance (Tursky, 1973), reporting stimulus intensity in units of delivered power removes any variability that is due to impedance differences among subjects. Thus, the stimulus intensities were programmed in voltage units, but the results are reported in power units.

At the end of testing, each subject was asked to check off all McGill Pain Questionnaire (MPQ) items (Melzack, 1975) that described their experience of the electrocutaneous stimuli. Regression analyses related these measures to weights in the INDSCAL subject space.

\section{Procedure}

Finger preparation and electrode placement. An area between the first and second phalanges of the left index and middle fingers was lightly abraded with fine sandpaper, cleaned with alcohol, and coated with Redux gel (Hewlett-Packard, Waltham, MA). If cuts or abrasions were present at one of these sites, the ring finger was substituted. Digital ring electrodes (TECA, Pleasantville, NY) were then secured to these prepared areas, taped to prevent drying of the gel, and insulated. If skin resistance exceeded $20 \mathrm{k} \Omega$, fingers were prepared again.

Ascending method of limits (AML). The subjects were familiarized with electrocutaneous stimuli through repeated pain threshold determinations. The subjects responded to increasing stimulus intensities (4-V steps), using the following intensity scale: no sensation, slight sensation, moderate sensation, strong sensation, uncomfortable, faint pain, moderate pain, and severe pain. In addition, when further increases in stimulus intensity were considered intolerable, the subject pressed a key labeled stop. Certain anchors were given: slight sensation was to be used when any sensation was detected; faint pain was used for anything stronger than just an uncomfortable feeling; and stop was used when higher intensities could not be tolerated. These determinations were repeated three times or until a stable intensity for tolerance was achieved.

Individual differences scaling (INDSCAL). The stimulus intensities were tailored to each subject's tolerance, resulting in subjectively equal intensities. Although uniform intensities across subjects are desirable, the use of the same intensities for all subjects would result in a highest intensity that would be too low for some subjects, who would not experience pain, and unbearable to others, who would refuse to participate. These intensities were determined by dividing the AML tolerance voltage into 16 equal parts. (For example, if stop was reported at $64 \mathrm{~V}$, the 1st stimulus would be $4 \mathrm{~V}$, the 2 nd stimulus $8 \mathrm{~V}$, etc., and the 16 th stimulus would be $64 \mathrm{~V}$.) 
Following practice trials, each subject rated the similarity of the 120 nonredundant stimulus pairs by using a 10-button linear keypad labeled not similar at one end and extremely similar at the other. (The number of nonredundant stimulus pairs is $n[n-1] / 2$, where $n$ stands for the number of stimuli.) As a check for consistency, 8 randomly selected stimulus pairs were repeated -4 toward the middle, and 4 toward the end of the presentation sequence. Two subjects (of the original 43) were excluded because of larger than average discrepancies between their two ratings.

These were the subjects' instructions:

\begin{abstract}
We wish to obtain judgments from you concerning how similar or dissimilar two electrical stimuli appear to be. After a tone, one stimulus of the pair will be presented, followed in 1-3 sec by another tone and the second stimulus. At the third tone, rate how similar these two electrical sensations appear to you by pressing one of the 9 buttons from "Not Similar" to "Extremely Similar." The intensities of the pairs are determined randomly and are of all possible intensity separations, so use as many different buttons as possible to rate the similarity of the two sensations.
\end{abstract}

Data were analyzed using the three-way multidimensional scaling program SINDSCAL (Pruzansky, 1975), a program designed to be an efficient implementation of the INDSCAL model. The INDSCAL model assumes that a set of $r$ factors or dimensions underlie the orientation of stimulus objects in a weighted Euclidean multidimensional space. Although the group stimulus space is common to all subjects, dimensions are expected to be variably important or salient to each individual. Individual differences are indexed by subject weights, which may be used to expand or contract the group dimension in proportion to its salience to the individual. Analysis begins with pairwise proximity judgments of stimulus objects, one matrix per subject, which are first converted to distances and then to a matrix of scalar products among stimuli (Torgerson, 1958). Scalar product matrices are normalized to unit variance by subject. Canonical decomposition of these matrices proceeds through an iterative process to simultaneously optimize, in the least squares sense, the subject weights and the distances between stimuli in $r$-dimensional space (Arabie et al., 1987; Carroll, 1972; Carroll \& Chang, 1970).

SINDSCAL solutions in up to six dimensions were generated, each from four different starting points. Examination of results from different starting points discloses whether the iterative routine converges to a local, rather than a desired global, minimum. The dimensionality of the accepted solution was determined according to the following criteria: A solution in $r$ dimensions was first accepted over the solution in $r-1$ dimensions if it substantially increased the model's goodness of fit to the scalar products matrix, specified in units of variance accounted for. The solution was next tested for interpretability; final acceptance rested on the demonstration of split-half reliability.

Property mapping (PREFMAP). After the INDSCAL procedure, the subject rated eight of the INDSCAL stimulus intensities (those numbered $2,4,6, \ldots, 16$ ) on each of 16 property scales. Only eight stimuli were used, in order to limit the task to an already arduous 128 trials. Following a tone, an electrical stimulus was delivered, and 1 of the 16 scales appeared on the computer screen. The subjects rated the stimuli (using a 10-point scale) on each of the scales in Table 2. A " 0 " indicated complete agreement with the property on the left side of the scale, and " 9 ," complete agreement with the property on the right. A random process determined which polar qualities appeared to the left or right of the scale, as well as the presentation orders of the electrical stimuli and of the property scales. The properties in Table 2 have been arranged so that stronger associations with dimensional ordering reflect more of the property to the right of the scale. PREFMAP data were collected only on Day 1.

The subjects received the following instructions:

In this study we wish to examine the meanings that these electrical stimuli have to you. Following a tone, a stimulus will be presented. You are to rate it from 0 to 9 on double-ended scales which will appear on the computer screen. An example of such a scale might be "Bad" at one pole and "Good" at the other. If you felt the stimulus to be "Extremely Bad," you would give it a 0 ; if "Somewhat Good," a 7 or 8; if neither good nor bad, then a 4 or 5 would be appropriate. In doing this task, please rate each stimulus on the basis of how it feels to you right now. There are no right or wrong answers. Also, try to respond with as many different response categories as possible.

Responses were analyzed using the PREFMAP2 program (Bell Laboratories, Murray Hill, NJ). PREFMAP models of increasing complexity were evaluated (Carroll, 1972; Carroll \& Arabie, 1980; Schiffman et al., 1981). In the vector (least complex) model, a multiple linear regression relates dimensional coordinates to property scale ratings. This model assumes that the dimensional ordering represents continuously increasing amounts of a property. More complex models did not fit better than the vector model, and are not presented.

Finally, the subjects were asked to check off all property scales not relevant to their experience of the stimuli, in order to identify the property scales that subjects did not use as bases for their similarity judgments.

\section{RESULTS}

\section{Stimulus Intensities}

The 16 stimulus intensities were based on each subject's tolerance. Figure 1 shows the mean power and associated sensory category applied to each of these stimuli on each test day. For Day 1 (filled circles), the lowest intensity averaged $0.8 \mathrm{~mW}$, whereas the most intense stimulus averaged $238.1 \mathrm{~mW}$. Note that error bars denote the $95 \%$ confidence limits both vertically, for response category, and horizontally, for stimulus power. (Although confidence limits do not allow inferences about ordinal scales like response category, we nevertheless find the vertical bars useful in describing the subjects' performance.) Given this caveat, then, on Day 1, for example, Stimulus 8 ranged between 40 and $70 \mathrm{~mW}$ for $95 \%$ of the sub-

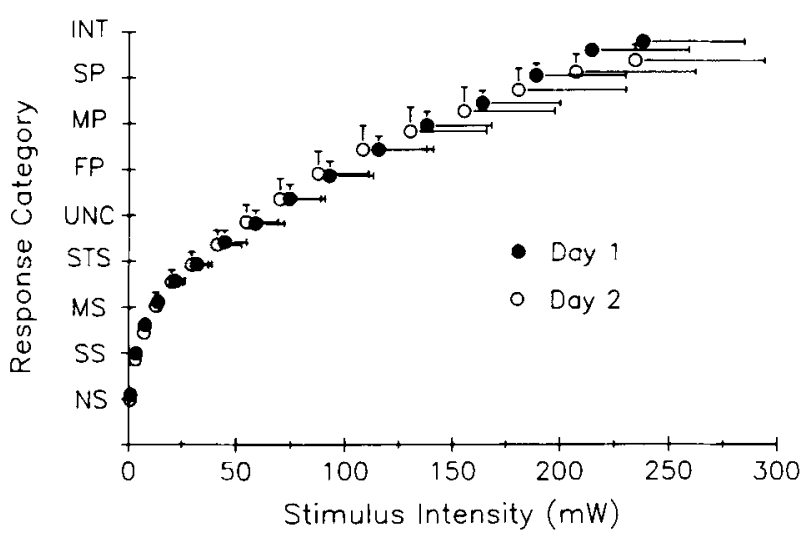

Figure 1. Average $(N=41)$ power and associated response category of the 16 stimuli used in the paired comparison INDSCAL procedure for Day 1 (open circles) and Day $2(N=25$; filled circles); bars indicate standard error of the mean both vertically (for response) and horizontally (for power). Abbreviations used: NS = no sensation; SLS = slight sensation; MS = moderate sensation; STS = strong sensation; UNC = uncomfortable; FP = fulnt pain; MP = moderate pain; SP = severe pain; INT = intolerable. 
jects, who labeled it uncomfortable, plus or minus approximately half a response category. Stimulus 16 ranged from 180 to $275 \mathrm{~mW}$ and produced average sensations between severe pain and intolerable. The 16 stimulus intensities determined on Day 2 (open circles) were statistically indistinguishable from those of Day 1.

\section{INDSCAL (Day 1 Data)}

Although not present when the subject made similarity judgments, sensory descriptors from the AML procedure were used to label objects in the group stimulus space as an aid to interpretation. Some of the 16 intensities receive the same label, since only 9 descriptors were used in the method of limits; all intensities were nevertheless distinguishable from one another.

Group stimulus space. On Day 1, a SINDSCAL solution in four dimensions produced a replicable and interpretable stimulus ordering. These dimensions accounted for $45 \%, 12 \%, 11 \%$, and $5 \%$ of variability in the scalar products matrix, a total of $73 \%$. Split-half reliability estimates were obtained by correlating, for each dimension, complementary stimulus coordinates from the two randomly selected group halves. These correlations were .99 , $.99, .96$, and .99 , respectively $(d f=13$, all $p s<.01)$.

By projecting points onto each axis in turn, dimensional interpretations are made from the placement of objects at the poles, as well as the entire order. Figure 2 shows the stimulus ordering on Dimensions 1 and 2. Dimension 1 , sensory magnitude (abscissa), was a continuous dimension, ordering stimuli quite well with respect to intensity, from $1.0 \mathrm{~mW}$, no sensation, at one pole, to $115.7 \mathrm{~mW}$, faint pain, at the other. Lower intensities were well separated, but higher, painful intensities, were clustered together. Dimension 2, pain intensity (ordinate), reflected pain-specific intensity judgments, since the highest intensity stimuli, 215.0 and $238.1 \mathrm{~mW}$, both labeled intolerable, and $189.2 \mathrm{~mW}$, severe pain, occupy one pole, and the lower intensity stimuli, 59.0-74.6 mW, labeled uncomfortable, occupy the other pole of this axis. This dimension also has a secondary (in the sense that it has a shorter extent), nonpainful attribute, which scales stimuli from uncomfortable to no sensation. These two dimensions clearly indicate an independent scaling of nonpainful and painful intensities.

Dimensions 3 and 4 are portrayed in Figure 3. Dimension 3 (abscissa), with stimulus intensities of 59.0 and $74.6 \mathrm{~mW}$, uncomfortable, at one pole and $3.6 \mathrm{~mW}$, slight sensation, at the other, suggests the nonpain intensity label. Similar to the sensory magnitude dimension, this dimension distinguished among a smaller range of intensities, omitting the no sensation intensity. Secondary attributes of pain intensity (uncomfortable to intolerable),

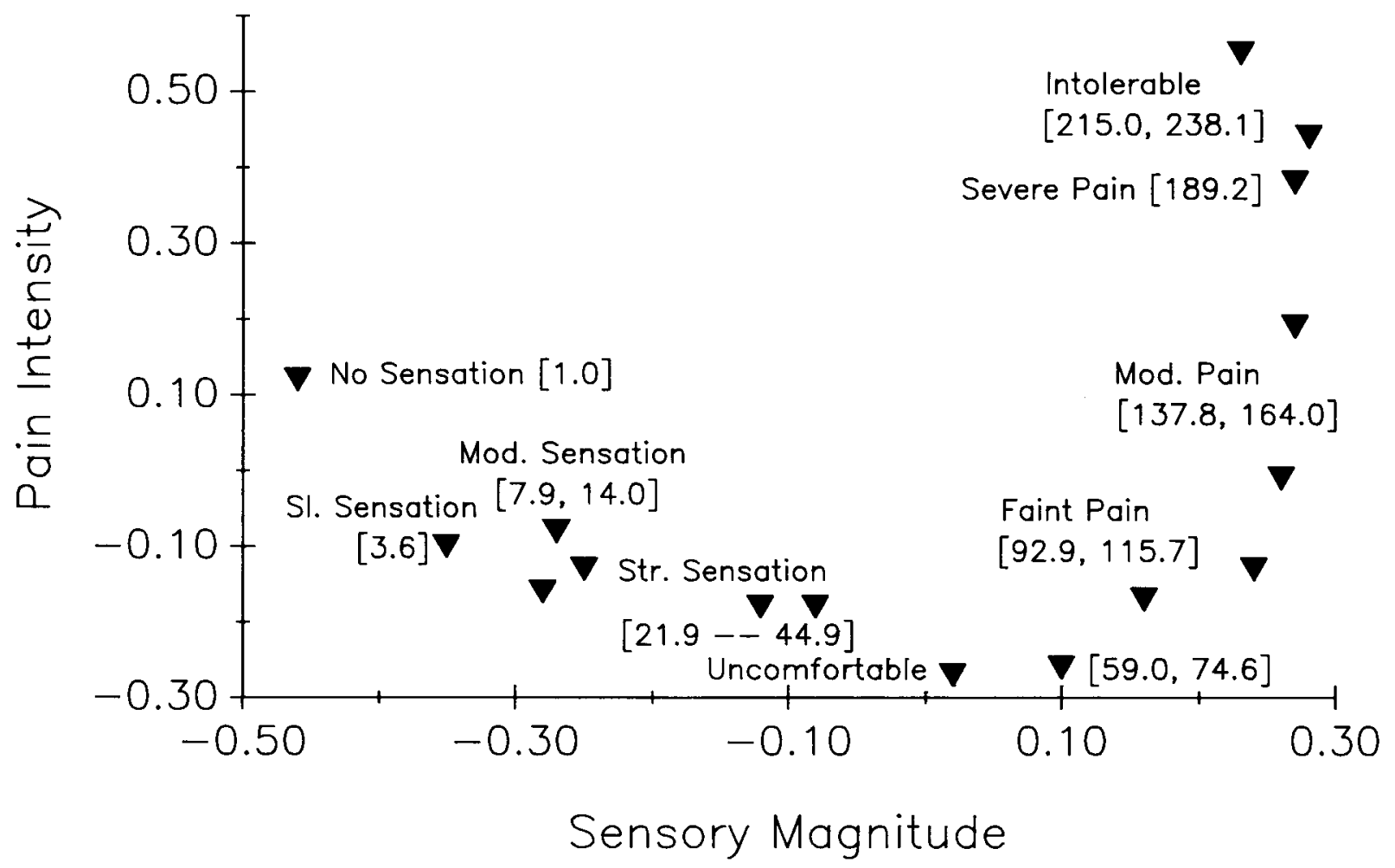

Figure 2. Sensory magnitude versus pain intensity dimensions. Two-dimensional plot of distances between the 16 electrical stimulus objects in normalized INDSCAL group stimulus space (Day $1, N=41$ ). Category labels refer to the mean response assigned to the stimulus in the ascending method of limits procedure (abbreviations as in Figure 1); numbers in brackets refer the mean power ( $\mathrm{mW}$ ) of that stimulus intensity. 


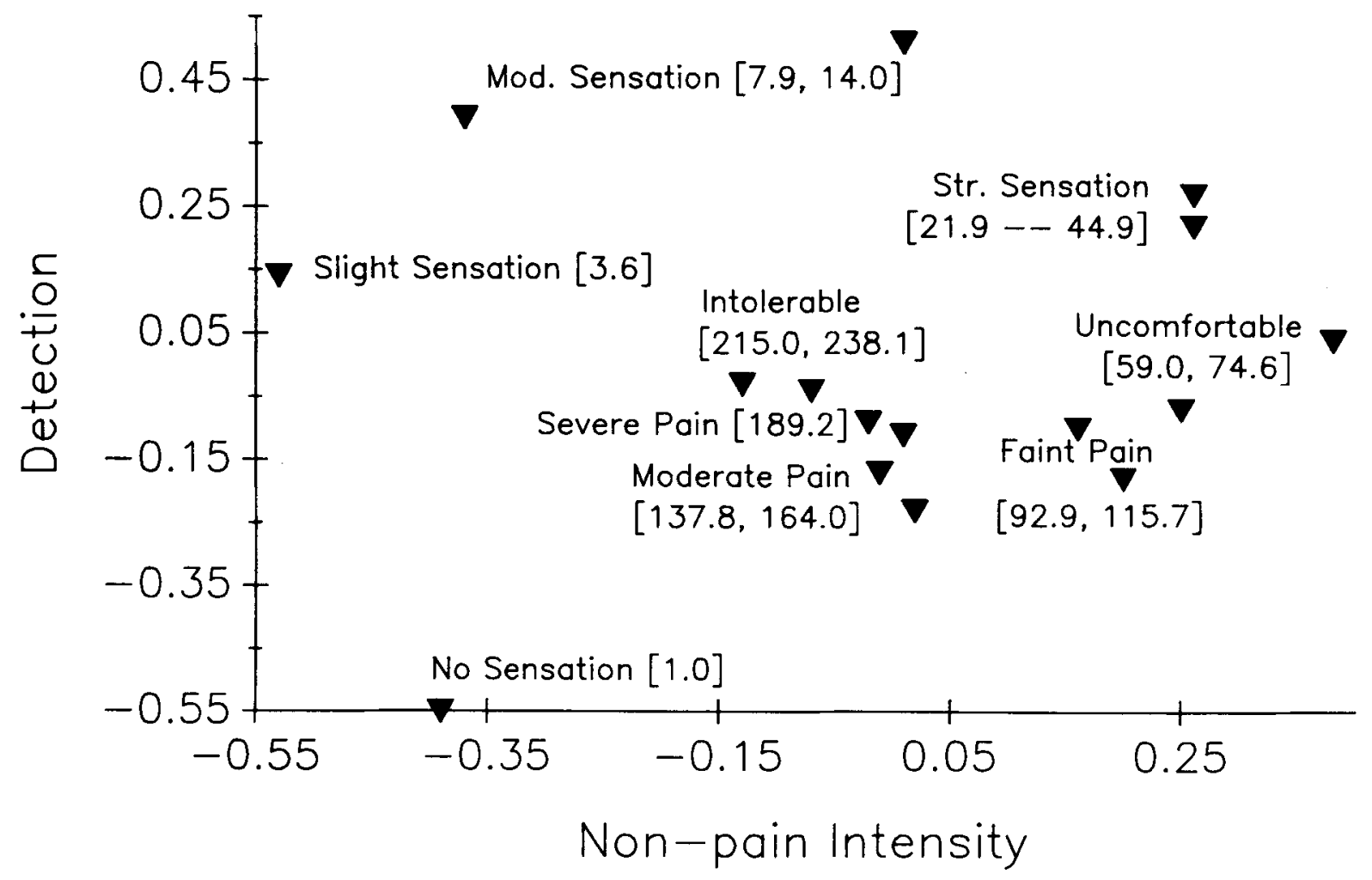

Figure 3. Nonpain intensity and detection dimensions. Labels as in Figure 2.

and of detection (slight sensation to no sensation) were also present. Dimension 4 (ordinate) positions the $1.0-\mathrm{mW}$ stimulus, no sensation, at one pole, isolated from the other stimuli, and most distant from the 7.9- and $14.0-\mathrm{mW}$ stimuli, labeled moderate sensation, at the other pole, suggesting the detection label.

Subject space. Subject (more generally, source) weights index individual differences in the salience of each of the group dimensions. The axes of this space are scaled in

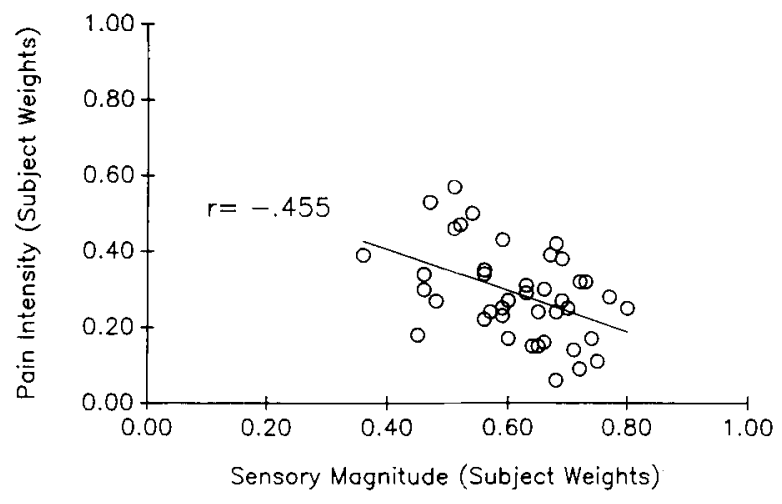

Figure 4. Two-dimensional representation of the subject space for the sensory magnitude and pain intensity dimensions. Note variability among subjects in the salience of these dimensions, and the inverse correlation between them, indicating that only one or the other dimension was used in making similarity judgments. units that are analogous to variance accounted for by that dimension; each point in this space represents an individual. In Figure 4, the subject weights are plotted for the sensory magnitude (abscissa) and pain intensity (ordinate) dimensions. The moderate negative correlation between these dimension weights suggests that as one dimension was used more, the other was used less.

Regression analyses. Examination of Figure 4 also shows that there was considerable variability among subjects in the use of each dimension. The relating of this variability to indices of psychological functioning is a potentially powerful technique for illuminating both dimensional meaning and the basis of individual differences. Forward-entry multiple linear regression (MLR) analyses (Cohen \& Cohen, 1983) were employed to relate subject weights on Dimensions 1 and 2 to each individual's use of the MPQ words. Subject weights on the sensory magnitude dimension were highest for subjects who frequently used the MPQ descriptors itchy, steady, and troublesome and rarely used intermittent or nagging to describe the electrical stimulation $[R=.72, F(5,35)=7.4, p<.001]$. Thus, this dimension was most salient to subjects endorsing sensory descriptors of the stimuli, supporting use of the sensory magnitude label. Subjects' weights on the pain intensity dimension were highest for those who frequently used the MPQ descriptors cruel or piercing and infrequently used continuous or splitting $[R=.66, F(4,36)=7.0, p<$ $.001]$. This dimension, therefore, was most salient to those who made affective evaluations of the stimuli, which sug- 
gests that the pain intensity dimension reflects affective more than sensory considerations. In general, these analyses indicate that individual differences in scaling reflect meaningful differences in stimulus experience.

\section{Comparison of Day 1 and Day 2 INDSCAL Results}

Data from the 25 subjects studied on both test days were combined in a single INDSCAL analysis, which yielded a four-dimensional space accounting for $72 \%$ of variability in the scalar products matrix. These dimensions ordered stimuli exactly as on Day 1, producing dimensions of sensory magnitude (variance accounted for, or VAF = 44\%); nonpain intensity (VAF $=15 \%$ ); pain intensity $(\mathrm{VAF}=8 \%)$; and detection (VAF $=5 \%)$. Strong betweenday reliability in stimulus ordering was indicated by the following correlations between complementary stimulus coordinates from separate day analyses: .96 (sensory magnitude); .76 (pain intensity); .87 (nonpain intensity); and .83 (detection).

Separate analyses of Day 1 and 2 data suggest that pain became a less important stimulus characteristic on Day 2, while nonpainful characteristics became more important. The pain intensity dimension accounted for $12 \%$ of variability on Day 1 and only 6\% on Day 2; values for the nonpain intensity dimension increased from $11 \%$ on Day 1 to $15 \%$ on Day 2 . There were no differences between days in either sensory magnitude or detection dimension weights. To test statistical differences between days, the average subject weights for each test day and dimension (from the joint-day analysis) were compared (Table 1). The nonpain intensity dimension showed a trend $[t(24)=$ $1.9, p<.10]$ toward higher weights and greater salience on Day 2 than on Day 1, while the pain intensity dimension showed an opposite trend $[t(24)=1.7, p<.15]$, toward higher weights on Day 1 than on Day 2. Thus, the data indicate a stable INDSCAL dimensional structure over time.

\section{PREFMAP}

In Table 2, the property scales are presented in order of variance explained by each dimension. Stimulus ordering on the sensory magnitude dimension was best matched by property vectors of increasing unpleasantness, fear, shame, and slowness. However, a total of nine scales (including sensory, affective, and arousal properties) accounted for substantial variability, suggesting that this dimension denotes a most general interpretation of magni-

Table 1

Mean Subject Weights (with Standard Deviations) for Each Test Day on Each Dimension of a Joint Dataset INDSCAL Solution $(N=25)$

\begin{tabular}{llllll}
\hline \multirow{2}{*}{\multicolumn{1}{c}{ Dimension }} & \multicolumn{2}{c}{ Day 1 } & & \multicolumn{2}{c}{ Day 2 } \\
\cline { 2 - 3 } \cline { 5 - 6 } \cline { 5 - 6 } Sensory magnitude & $M$ & $S D$ & & $M$ & $S D$ \\
Pain intensity & .57 & .11 & & .57 & .12 \\
Nonpain intensity & .26 & .11 & & .22 & .08 \\
Detection & .31 & .09 & & .35 & .11 \\
\hline
\end{tabular}

Table 2

PREFMAP Summary Indicating the Proportion of Variance Accounted for, and the Percentage of Subjects Whose Data Were Fit Reliably $(p<.01)$, by Each of the 16 Property Scales in the Four-Dimensional INDSCAL Space

\begin{tabular}{|c|c|c|c|c|c|}
\hline \multirow[b]{2}{*}{ Property Scale } & \multicolumn{4}{|c|}{ Variance Accounted For } & \multirow{2}{*}{$\begin{array}{c}\% \\
\text { Subjects }\end{array}$} \\
\hline & Dim 1 & Dim 2 & Dim 3 & Dim 4 & \\
\hline Pleasant-unpleasant & $.97^{*}$ & 0 & 0 & 0 & 54 \\
\hline $\begin{array}{l}\text { Socially accep- } \\
\text { table-shameful }\end{array}$ & $.94^{*}$ & .02 & .02 & .01 & 46 \\
\hline Fast-slow & $.91^{*}$ & .03 & 0 & 0 & 35 \\
\hline Lethargic-aroused & $.90^{*}$ & .01 & .02 & 0 & 32 \\
\hline Confident-anxious & $.87^{*}$ & .11 & 0 & .01 & 32 \\
\hline Reassuring-frightening & $.84 *$ & .01 & 0 & .11 & 51 \\
\hline Weak-strong & $.71^{*}$ & .26 & .02 & 0 & 59 \\
\hline Safe-dangerous & $.70^{*}$ & .24 & .04 & .01 & 60 \\
\hline Localized-pervasive & $.59 *$ & .28 & .12 & 0 & 37 \\
\hline Surface-deep & .04 & $.66^{*}$ & .18 & .09 & 44 \\
\hline Optimistic-pessimistic & .27 & $.64^{*}$ & .04 & .03 & 16 \\
\hline No pain-worst pain & 16 & $.57^{*}$ & .16 & .11 & 54 \\
\hline Brief-lasting & .43 & .43 & $: 12$ & .01 & 32 \\
\hline Soothing-irritating & .41 & .39 & .01 & .19 & 41 \\
\hline In control-helpless & .05 & .32 & 0 & .36 & 35 \\
\hline Hot-cold & .35 & .03 & .04 & .10 & 41 \\
\hline
\end{tabular}

${ }^{*} p$ (variance accounted for $\left.=0\right)<.01$.

Table 3

Percent of Subjects Rating a Property Scale as Relevant to Their Similarity Judgments $(N=41)$

\begin{tabular}{lc}
\multicolumn{1}{c}{ Scale } & \% Relevant \\
\hline No pain-worst pain & 98 \\
Weak-strong & 98 \\
Pleasant-unpleasant & 95 \\
Soothing-irritating & 93 \\
Surface-deep & 90 \\
Brief-lasting & 90 \\
In control-helpless & 90 \\
Composed-anxious & 88 \\
Reassured-frightened & 88 \\
Local-pervasive & 85 \\
Fast-slow & 85 \\
Lethargic-aroused & 80 \\
Safe-dangerous & 80 \\
Hot-cold & 61 \\
Optimistic-pessimistic & 54 \\
Socially acceptable-shameful & 24 \\
\hline
\end{tabular}

tude, rather than the magnitude of a specific stimulus property. Dimension 2 , by contrast, was specifically related to properties of pain, pessimism, and depth, supporting the narrow interpretation of this dimension as one reflecting pain intensity. None of the property scales chosen showed significant associations with either the nonpain intensity or the detection dimension.

The percentage of subjects who rated each property scale as relevant to their similarity judgments is presented in Table 3. Concordance was generally found; property scales predicted by dimensional ordering in PREFMAP were also chosen as relevant to INDSCAL similarity judgments. Thus, for example, no pain-worst pain was strongly related to the pain intensity dimension, and $98 \%$ of the subjects rated this property as relevant to making their 
similarity judgments. Similarly, hot-cold was unrelated to any dimension and was not chosen as relevant to similarity judgments. However, although scales of socially acceptable-shameful and pessimistic-optimistic were notably less relevant than the other scales, PREFMAP analysis showed them to be significantly related to the INDSCAL dimensions. This suggests that PREFMAP can produce "false alarms," reliably relating stimulus objects to property scales when these properties were not cognitively "active" during the INDSCAL task.

\section{DISCUSSION}

Although the electrical stimuli were varied on a single intensity dimension, analysis indicated that subjects perceive the four distinct intensity-related qualities discussed below.

The sensory magnitude dimension was characterized by a continuous ordering of stimuli from the lowest intensity to the faint pain threshold. PREFMAP analyses suggested that magnitude was scaled in a very general sense, since sensory, affective, and arousal property scales were all related to this dimension. Regression analyses, however, indicated that sensory characteristics were most important, since this dimension was most salient to subjects who chose MPQ sensory descriptors to describe their experience of the stimuli.

The pain intensity dimension scaled the highest intensity stimuli continuously from pain threshold (uncomfort$a b l e$ ) to withdrawal. PREFMAP analyses supported the use of this label, since only this dimension was associated with the no pain-worst possible pain property scale. Surface-deep and optimistic-pessimistic property scales were also related to this stimulus ordering, suggesting that this dimension reflected judgments of both sensation and affect. Regression analyses confirmed the importance of both sensation and affect to the judgments, since subjects with the highest weights on this dimension frequently described the stimuli as "cruel" and "piercing." Thus, although affective "stimuli" were not presented, affective responses are related to the use of this dimension.

Dimensions 1 and 2 produce independent scales of painful and nonpainful sensations. Dimension 1 allows fine discrimination only among intensities as great as faint pain, whereas Dimension 2 allows fine discrimination among the more painful intensities, closely following what one would expect from the activity of large- and smalldiameter peripheral nerve fibers, respectively. Initial and continuing activity of low-threshold, large-diameter fibers could underlie the scaling of nonpainful stimuli; after their thresholds are reached, smaller diameter fibers then provide information for distinguishing among increasingly painful stimulus intensities. These data are consistent with several magnitude estimation studies of electrocutaneous stimulation, which suggest that nonpainful sensations and pain are mediated by separate processes. Rosner and Goff (1967), for example, reported steeper power functions for nonpainful than for painful stimuli. More recently,
Higashiyama and Tashiro (1989) have also reported different power function exponents for judgments in the painful and nonpainful stimulus intensity ranges; furthermore, response adaptation occurred over repeated blocks of nonpainful, but not painful, stimulus trials. The present data are also consistent with the results of Clark et al. (1986), who found separate painful and nonpainful dimensions underlying judgments of thermal cutaneous stimuli. Thus, pain stimuli appear to be judged on their own continuum, not on the upper end of a single sensory strength dimension.

The nonpain intensity dimension, with slight sensation at one pole and strong sensation at the other, reflects the nonpainful aspects of the sensory experience, minimizing painful or just detectable stimulus properties. Fortunately, the INDSCAL analysis is clear, since none of the PREFMAP scales chosen was predicted by this dimension. Inclusion of a scale such as slight-moderate, which reflects the experience of moderate intensity stimuli, might prove to be a good correlate of this ordering. Although it is similar to sensory magnitude in scaling nonpainful sensory qualities, this dimension spans a narrower stimulus range. Furthermore, regression analyses indicate that this dimension was uniquely important to a group of subjects who frequently selected wrenching and heavy MPQ descriptors.

The detection dimension, which segregates no sensation from moderate and strong sensations, reflects the distinction between nothing and perceptible sensations of any type. None of the properties sampled were related to this dimension. Like Dimension 3, then, the relationship of other property scales to this dimension-for example, nothing-something or uncertain-certain-must be explored. This dimension, like the pain intensity dimension, was more salient to subjects who frequently chose affective descriptors from the MPQ.

Thus, each dimension was defined by its particular focus on a segment of the physical intensity order. The scaling of stimuli on Dimension 1 was easiest to interpret, since the lower intensity stimuli that defined the dimension were well separated from one another, whereas more painful, nondefinitional, stimuli were clustered closely together at one pole (see Figure 2). Interpreting Dimensions 2-4 was somewhat more difficult, because major and minor attributes (Kruskal \& Wish, 1978) were present. For instance, although Dimension 2 was clearly defined by polar objects of uncomfortable and intolerable, indicating a pain intensity label, a nonpain attribute from uncomfortable to no sensation was also present. When these dimensions are "unfolded" (Coombs, 1964), however, simple monotonic scales are revealed. In this instance, unfolding Dimension 2 (Figure 2) at the uncomfortable "ideal point" results in a scale composed of two line segments. The longer segment maximally discriminates among progressively more painful stimuli, indicating the pain intensity label, while the shorter segment scales, with lesser discriminability, the nonpainful stimuli. The reverse is true of Dimension 3 (Figure 3), where unfolding at the strong sensation point results in a longer nonpainful line segment and the nonpain intensity label. Stimulus proper- 
ties that are less important to the definition of a dimension are apportioned less distance on that dimension, and in a limiting case such as Dimension 1, they are clustered together so as to cover virtually no distance.

Although the nonpain intensity and detection dimensions were interpretable, they were not validated by the PREFMAP analyses. Since they satisfied criteria of reliability and variance accounted for, however, we hypothesize that they are "real." Because they were unexpected, however, appropriate property scales were not present to detect them. Data from an independent sample replicated the four dimensions reported here (Janal, Clark, \& Clark, 1990), indicating that they are robust, and additional property mapping studies are underway to validate the present interpretation. It has not been ruled out, however, that the third and fourth dimensions could be functions simply of individual differences in use of the response scale (which could, in turn, be systematically related to the subjects' use of the MPQ descriptors). Further research is needed to determine whether these dimensions are "real" or artifactual. To determine whether they are physiologically "real," it will be necessary to manipulate the physical correlates of these dimensions. If these dimensions are validated, they will expand the number of dimensions thought to underlie the perception of intensity.

Both split-half and retest reliability measures indicate a stable group stimulus space. Although split-half reliability estimates have commonly been determined, this study is the first to show that INDSCAL dimensions are stable over a 2-week period. In consistency with the reliability data, average subject weights did not differ significantly on the 2 days. This demonstration encourages the development of clinical scales based on the INDSCAL model, in which it is desirable to compare different patients, as well as the same patient under different treatments, on a common set of scales.

Stimulus objects in the Dimension 1 and 2 space describe a semicircle. This pattern raises the question of whether this nonlinearity reflects a single "bent" dimension ("horseshoe effect"; Shepard, 1974), or two valid dimensions. In defense of the latter, we would argue that: (1) Artifactual extra dimensions are not likely to occur with three-way MDS analyses such as INDSCAL because the use of individual data provides dimensional uniqueness (Clark et al., 1986). (2) The presence of an interpretable (unrotated) solution also suggests the absence of artifact. If Dimension 2 were an artifact, subject weights would not be related to a different set of MPQ responses from that which was related to Dimension 1. (3) A onedimensional solution failed to correctly order (i.e., in order of increasing intensity) either the lowest or the highest stimulus intensities; to do this, a solution in at least two dimensions is necessary. Future work can provide a strong test of the necessity of two dimensions by manipulating judgments along one dimension at a time. For example, a differential nerve block of myelinated (nonpain) fibers, such as ischemic occlusion of the arm, would be expected to reduce subject weights on Dimension 1 more than on Dimension 2.

Significant relationships between dimensions and property scales have been interpreted to mean that that property was cognitively active when subjects made similarity judgments. Results from this study suggest that this is not always true. Scales like hot-cold were not related to any dimension and were not endorsed as relevant. Similarly, scales rated as relevant were strongly related to a dimension. However, some scales were significantly associated with a dimension but were not endorsed as relevant to similarity judgments. Socially acceptable-shameful, for example, was strongly related to Dimension 1, but was found to be irrelevant by $76 \%$ of the sample. Although subjects are able to rate stimuli on these property scales when asked to, this does not necessarily mean that that property was cognitively active when the subject judged similarities. Since such activity is necessary for one to infer dimensional meaning from property mapping data, PREFMAP instructions should request that the subject choose the property scales that were active when they made their similarity judgments, and judge stimuli only on these scales. Alternately, the subject could rate how important each property was to his or her INDSCAL judgments according to a procedure similar to that used above. These ratings could then be incorporated as weights in the regression equation.

Previous work had indicated the presence of independent painful and nonpainful intensity dimensions. Present results confirm these separate dimensions and suggest the presence of two additional nonpain dimensions. These INDSCAL analyses produced reliable measures of both sensory and affective responses to laboratory pain stimulation, suggesting that the model is well suited to the study of clinical pain.

\section{REFERENCES}

Algom, D., RAphaeli, N., a Cohen-RAz, L. (1986). Integration of noxious stimulation across separate somatosensory communication systems: A functional theory of pain. Journal of Experimental Psychology: Human Perception \& Performance, 12, 92-102.

Arabie, P., Carroll, J. D., DeSarbo, W. S. (1987). Three-way scaling and clustering (Quantitative Applications in the Social Sciences 07-065). Newbury Park, CA: Sage.

Carasso, R. L., Yehuda, S., \& Mostofsky, D. I. (1984). Multidimensional scaling of pain experiences. Bulletin of the Psychonomic Society, 22, 349-350.

CARroll, J. D. (1972). Individual differences and multidimensional scaling. In R. N. Shepard, A. K. Romney, \& S. Nerlove (Eds.), Multidimensional scaling: Theory and applications in the behavioral sciences: Vol 1. Theory (pp. 105-155). New York: Seminar Press.

Carroll, J. D., \& Arabie, P. (1980). Multidimensional scaling. In M. R. Rosenzweig \& L. W. Porter (Eds.), Annual review of psychology: Vol. 3 I (pp. 607-649). Palo Alto, CA: Annual Reviews.

Carroll, J. D., Chang, J. J. (1970). Analysis of individual differences in multidimensional scaling via an $N$-way generalization of “Eckart-Young"' decomposition. Psychometrika, 35, 283-319.

Clark, W. C. (1974). Pain sensitivity and the report of pain: An introduction to sensory decision theory. Anesthesiology, 40, 272.287. Clark, W. C., Carroll, J. D., Yang, J. C., Janal, M. N. (1986). 
Multidimensional scaling reveals two dimensions of thermal pain. Journal of Experimental Psychology: Human Perception and Performance, 12, 103-107.

Clark, W. C., Ferrer-Brechner, T., Janal, M. N., Carroll, J. D., * YANG, J. C. (1989). The dimensions of pain: A multidimensional scaling comparison of cancer patients and healthy volunteers. Pain, 37, 25-32.

Clark, W. C., Janal, M. N., \& Carroll, J. D. (1989). Multidimensional pain requires multidimensional scaling. In C. R. Chapman \& J. D. Loeser (Eds.), Issues in pain measurement (pp. 285-325). New York: Raven Press.

COHEN, J., \& COHEN, P. (1983) Applied multiple correlation/regression analysis for the behavioral sciences. Hillsdale, NJ: Erlbaum.

CoOMBs, C. H. (1964). A theory of data. New York: Wiley.

Gracely, R. H., McGrath, P., \& Dubner, R. (1978). Ratio scales of sensory and affective verbal pain descriptors. Pain, 5, 5-18.

HigashiYama, A., Tashiro, T. (1989). Magnitude estimates for electrical pulses: Evidence for two neural mechanisms. Perception \& Psychophysics, 45, 537-549.

Janal, M. N., Clark, W. C., \& Clark, S. B. (1990, April). Effects of anxiety on judgments of noxious electrocutaneous stimulation: $A$ multidimensional scaling (MDS) analysis. Paper presented at the meeting of the International Association for the Study of Pain, Adelaide, Australia.

KRUSKAL, J. B., WISH, M. (1978). Multidimensional scaling (Quantitative Applications in the Social Sciences 07-001). Beverly Hills, CA: Sage.

Kwilosz, D. M., Green, B. F., * Torgerson, W. S. (1983, October). Qualities of hurting: The language of pain. Paper presented at the meeting of the American Pain Society, Chicago.

Melzack, R. (1975). The McGill Pain Questionnaire: Major properties and scoring methods. Pain, 1, 277-299.

MORLEY, S. (1989). The dimensionality of verbal descriptors in Tursky's pain perception profile. Pain, 37, 41-50.
PruZanSKY, S. (1975). How to use SINDSCAL: A computer program for individual differences in multidimensional scaling. Unpublished manuscript, AT\&T Bell Laboratories, Murray Hill, NJ.

RosNer, B., \& GoFf, N. R. (1967). Electrical responses of the nervous system and subjective scales of intensity. In W. D. Neff (Ed.), Contributions to sensory physiology (Vol. 2, pp. 169-221). New York: Academic Press.

Schiffman, S. S., Reynolds, M. L., \& Young, F. W. (1981). Introduction to multidimensional scaling. New York: Academic Press.

SHEPARD, R. N. (1974). Representation of structure in similarity data: Problems and prospects. Psychometrika, 39, 373-421.

SHEPARD, R. N. (1980). Multidimensional scaling, tree-fitting, and clustering. Science, 210, 390-398.

Stevens, S. S., Carton, A. S., \& Schickman, G. M. (1958). A scale of apparent intensity. Journal of Experimental Psychology, 56, 328-334.

TORGERSON, W. S. (1958). Theory and methods of scaling. New York: Wiley.

Torgerson, W. S., \& DenBebBa, M. (1983). The structure of pain descriptors. In R. Melzack (Ed.), Pain measurement and assessment (pp. 49-54). New York: Raven Press.

TuRsky, B. (1973). Physical, physiological and psychological factors that affect pain reaction to electric shock. Psychophysiology, 11, 95-112.

Tursky, B., Jamner, L. D., \& Friedman, L. (1982). The Pain Perception Profile: A psychophysical approach to the assessment of pain report. Behavior Therapy, 13, 376-394.

W ACK, J. T., \& TURK, D. C. (1984). Latent structure of strategies used to cope with nociceptive stimulation. Health Psychology, 3, 27-43.

(Manuscript received January 22, 1990 revision accepted for publication March 1, 1991.) 